\title{
Neuropsychological rehabilitation of memory deficits and activities of daily living in patients with Alzheimer's disease: a pilot study
}

R. Ávila, C.M.C. Bottino, I.A.M. Carvalho, C.B. Santos,

C. Seral and E.C. Miotto
Projeto Terceira Idade, Instituto de Psiquiatria, Faculdade de Medicina, Universidade de São Paulo, São Paulo, SP, Brasil
Correspondence

R. Ávila

Rua Guarara, 529, Cjto. 135

04523-015 São Paulo, SP

Brasil

Fax: +55-11-3061-2208

E-mail: avilarenata@uol.com.br

Research supported by FAPESP (No. 01/08189-8).

Received October 16, 2003

Accepted July 19, 2004

\begin{abstract}
Patients with Alzheimer's disease (AD) gradually lose their cognitive competence, particularly memory, and the ability to perform daily life tasks. Neuropsychological rehabilitation is used to improve cognitive functions by facilitating memory performance through the use of external aids and internal strategies. The effect of neuropsychological rehabilitation through memory training - motor movements, verbal association, and categorization - and activities of daily living (ADL) training was tested in a sample of 5 elderly out-patients (mean age: $77.4 \pm 2.88$ years), with mild AD (Mini-Mental State Examination score: $22.20 \pm 2.17)$ and their caregivers. All patients had been taking rivastigmine $(6-12 \mathrm{mg} /$ day) for at least 3 months before being assigned to the rehabilitation sessions, and they continued to take the medication during the whole program. Just before and after the 14-week neuropsychological rehabilitation program all patients were assessed by interviewers that did not participate in the cognitive training, using the Mini-Mental State Examination, Montgomery-Alsberg Depression Rating Scale, Hamilton Anxiety Scale, Interview to Determine Deterioration in Functioning in Dementia, Functional Test, Memory Questionnaire of Daily Living for patient and caregiver, Quality of Life Questionnaire for patient and caregiver, and a neuropsychological battery. The results showed a statistically significant improvement in ADL measured by Functional Test $(\mathrm{P}=0.04)$, and only a small improvement in memory and psychiatric symptoms. Our results support the view that weekly stimulation of memory and training of ADL is believed to be of great value in $\mathrm{AD}$ treatment, not only delaying the progress of the disease, but also improving some cognitive functions and $\mathrm{ADL}$, even though $\mathrm{AD}$ is a progressively degenerative disease.
\end{abstract}

Key words

- Alzheimer's disease treatment

- Neuropsychological rehabilitation

- Memory

- Daily living activities

\section{Introduction}

Alzheimer's disease (AD) is a progressive dementia in which memory deficit is one of the earliest and most pronounced symptoms (1). As the disease progresses, other cognitive functions such as language and general intellectual performance also become impaired. This decline in cognitive function has additional effects. For example, cognitive status is highly correlated with caregiver burden and functional impairment. 
Both of these factors influence daily living activities, the ability of the patients to live alone and, of course, their quality of life. Patients become insecure about performing simple daily living tasks such as using the telephone, paying bills, going out and returning home alone, and dressing themselves properly.

At the neuropathological level, $\mathrm{AD}$ is associated with the development of plaques and neurofibrillary tangles within the brain. Due to an increase in life expectancy for our population and its cost to society in terms of nursing and medical care and human suffering, the impact of senile dementia is substantial. Beyond these social issues, there is a considerable current interest in the possibility of alleviating dementia symptoms and reducing the progression of the cognitive decline, which is one of the most dramatic symptoms of the illness.

Neuropsychological rehabilitation (NR) is a new field of research and as such requires further development in specific areas including more efficient measures for assessment and outcome evaluation. In addition, there should be more accurate documentation regarding the effectiveness of NR methods for AD patients, a better understanding of the factors that influence the outcome of intervention, clearer evidence of both effectiveness and longer-term impact and gains, and a commitment to ensure that effective intervention is disseminated and implemented in standard clinical settings (2).

Current research in this area is recognizing the relevance of NR for people with dementia (3). Although papers have been published on this issue, with new developments and recent findings, researchers have usually been testing a single technique to rehabilitate demented patients (4-6), whereas in the present NR study a combination of cognitive techniques was used. The present study was based on the definition of NR as "a process of active change aimed at enabling people, who are disabled by injury or disease, to achieve an optimal level of physical, psychological, and social function" (7). NR can maximize functioning across a whole range of areas including physical health, psychological well being, daily living skills, and social relationships (8). Moreover, NR applied to AD patients aims to optimize functions, minimize excessive disability risk and prevent the development of negative social psychology (9).

The early identification of the disease is crucial for better treatment results since there are more preserved cognitive functions to work with in the early stages. The work with neurodegenerative diseases like $\mathrm{AD}$ involves a psychosocial approach directed at the needs of patients and caregivers. Therefore, an effective interview and assessment should address the patient's life style, socioeconomic background, as well as functional, psychological and cognitive features. In this context, NR provides a framework for a multidisciplinary treatment of AD.

NR is not static and all treatment strategies will depend on the severity of the specific characteristics and environment of $\mathrm{AD}$ patients. The advent of drug treatment for AD patients emphasizes the need for NR. Combined cognitive and pharmacological approaches have been explored, showing promising results as a helpful strategy for $\mathrm{AD}$ patients and their caregivers (10). The objective of the present study is to report on the tests and scales used to evaluate and reevaluate cognitive status, the efficiency of implicit and explicit memory techniques and activities of daily living (ADL) training of patients with $\mathrm{AD}$ in NR programs.

\section{Subjects and Methods}

\section{Subjects}

After the protocol was approved by the Ethics Committee and written informed consent was signed by each patient, 5 mildly impaired probable $\mathrm{AD}$ patients, diagnosed 
according to ICD-10 and NINCDS-ADRDA criteria and having used rivastigmine, 6 to 12 $\mathrm{mg}$ /day, for more than 3 months, started an NR training program. All subjects were classified as mildly ill on the basis of Clinical Global Impression.

Just before and after the 14-week period of cognitive training, all patients were evaluated using the Mini-Mental State Examination (11), Montgomery-Alsberg Depression Rating Scale (12), Hamilton Anxiety Scale (13), Interview to Determine Deterioration in Daily Functioning in Dementia (14), Functional Test (Ávila R, unpublished data; see Appendix), Memory Questionnaire of Daily Living (15) for patient and caregiver, Quality of Life Questionnaire (16) for patient and caregiver, and neuropsychological test battery. Neuropsychological test battery consisted of the Wechsler Memory Revised Scale (17), Wechsler Intelligence Revised Scale (18), Fuld Object Memory Evaluation (19), Recognition Memory Face (20), Boston Naming Test (21), and Verbal Fluency Semantic (animals) $(22,23)$ and Phonemic (24).

The medical condition and socio-economic and demographic characteristics of the patients are presented in Table 1.

\section{Memory training program}

The modality-specific memory rehabilitation works better using the intact modality to support the impaired one (25). Since AD patients have their implicit memory almost intact at the onset of the disease, it seems to be the best modality to compensate for explicit memory deficits. This work can be done using emotional and perceptual learning, priming, motor skills, habits, conditioning, and categorization (26). Explicit memory can also be worked on, especially with external aids and with Errorless Learning technique (27).

In the present study, motor movements, verbal association and categorization were applied to increase both learning and memory.

Motor movements. In order to learn a colleague's name, all patients introduced themselves by name and mentioned their hobbies or interests. Patients were instructed to choose a particular motor movement that matched each hobby, like moving fingers to play the piano. This movement should be associated with the person's name and face. At the beginning of each group session a motor movement mimic representing someone's hobby was associated with the person's name. Before recalling his/her name, patients were encouraged to recall his/her hobby. If they could not remember the hobby, movement or name cues were presented.

Verbal association. In order to improve learning and memory for words, patients were asked to create a sentence or a short story with the words intended to be learned or remembered. Each sentence should be constructed in such a way as to evoke a great deal of emotion. Patients were encouraged to remember the sentence and the words. If they could not remember alone, cues were presented like in a recognition test.

Categorization. To improve learning and memory for words the following exercise was given to each patient. First, a list of words was presented to the patients. They were then asked to divide the list into categories (clothing, food, etc.). In order to recall

Table 1. Clinical and sociodemographic characteristics of patients with Alzheimer's disease before cognitive rehabilitation.

\begin{tabular}{lc}
\hline Variable & Patients $(\mathrm{N}=5)$ \\
\hline Gender & Female $=4$ \\
& Male $=1$ \\
Age (years) & $77.4 \pm 2.88(73-80)$ \\
Schooling (years) & $6.60 \pm 2.32(2-15)$ \\
Marital status & Married: 2 \\
& Widowed: 3 \\
MMSE & $22.20 \pm 2.17(20-25)$ \\
ADL & $42.80 \pm 4.55(38-47)$
\end{tabular}

Data are reported as means $\pm S D$, with the range in parentheses. MMSE = Mini-Mental State Examination; $A D L=$ interview to determine deterioration in daily functioning in dementia. 
the list, they were asked to remember the categories.

\section{ADL training}

For this procedure, functional tasks were used in which patients were trained in the following four activities: telephone use, giving and receiving messages, diary use, and steps to prepare a sandwich. As AD patients have difficulty in transferring spontaneously a learned technique to an actual day-to-day problem, daily living situations were simulated. For example, in order to work on phone skills or receiving messages and taking notes, the training was done using a telephone, paper and pen, simulating a phone call. Similarly, when learning to write appointments in a dairy, a diary was used with real appointments.

\section{Support intervention}

Group support intervention was provided for caregivers as well, because of the reduction in the quality of life and increase in the depressive symptoms among caregivers (28). The caregivers attended a monthly group session focusing on orientation about the course of $\mathrm{AD}$ and its prognosis, counseling and support. All participants were encouraged to share their experiences and any coping strategies. The caregivers were always instructed to do some activities with the patients as homework.

\section{Group and individual sessions}

NR consisted of 60-min weekly group sessions and 30-min weekly individual sessions focusing on implicit and explicit memory training, temporal and spatial orientation, language abilities, developing compensatory strategies (for daily living deficits) and training for ADL, associated with social interaction. The same program was used in both group and individual sessions, but in the individual sessions more attention was given to specific patient difficulties. For example, one patient was able to utilize the aid of a diary very well, but she had great difficulty in using the telephone. Intensive phone training was done in her individual sessions, while the use of the diary was just reinforced.

The Errorless Learning technique described by Baddeley and Wilson (27) was applied throughout the program to enhance each patient's correct procedures and to avoid memorization of the wrong pattern. Throughout the training many facilitating clues were given to the patients, and as the activities became easier for them, fewer clues were given and so on until no clues were necessary. This is because patients with episodic memory deficit are not capable of remembering their mistakes and therefore cannot correct them. Thus, they do not learn from their mistakes as people without such a deficit do. Therefore, it becomes fundamental that learning should occur in a facilitating manner, always driving for the correct procedure.

\section{Statistical analysis}

The continuous scores of the cognitive tests and scales pre- and post-treatment were compared using the Wilcoxon test. The effect size (ES) was calculated according to the following formula:

$\mathrm{ES}=\frac{\text { mean post-treatment }- \text { mean pre-treatment }}{\text { pre-treatment standard deviation }}$

Rockwood et al. (29) stated that ES takes into account the within-group variance in performance at baseline and that a high number indicates a greater therapeutic effect.

Data were analyzed statistically using the SPSS 9.0 software for Windows.

\section{Results}

Table 2 shows the results of the scales applied to patients and caregivers before and 
after NR. Although there was only statistically significant difference between pre- and post-treatment in the Functional Test $(\mathrm{P}=$ 0.04 ), the group revealed a modest improvement after treatment in all scales, with the exception to the MDLQ patient.

Although Table 3 does not show significant differences in neuropsychological evaluation between pre- and post-treatment, most tests revealed a modest improvement in per- formance for patients, except for Verbal Fluency and Backward Digit Span. This improvement was particularly noted in memory tests. Table 4 shows the ES of some tests and scales, where only small positive effect could be noted, except in Functional Test, where a significant ES was obtained ( $\mathrm{ES}=1.00$ ).

These results are consistent with the NR program, which emphasize memory and ADL training.

Table 2. Results of the scales applied to patients and caregivers pre- and post-neuropsychological rehabilitation (NR).

\begin{tabular}{lccc}
\hline \multicolumn{1}{l}{ Scale } & \multicolumn{1}{c}{ Pre-NR } & Post-NR & Statistics \\
\hline MMSE & $22.20 \pm 2.17(20-25)$ & $23.80 \pm 5.22(16-29)$ & $Z=-0.31 ; P=0.75$ \\
HAM-A $^{+}$ & $7.60 \pm 5.41(4-16)$ & $2.80 \pm 4.09(0-9)$ & $Z=-1.48 ; P=0.13$ \\
MADRS $^{+}$ & $5.60 \pm 8.41(0-20)$ & $4.80 \pm 6.42(0-16)$ & $Z=-0.10 ; P=0.91$ \\
QOL - Patient & $37.60 \pm 6.91(29-48)$ & $42.00 \pm 7.38(33-51)$ & $Z=-0.41 ; P=0.67$ \\
QOL - Caregivers & $31.60 \pm 5.64(23-37)$ & $37.00 \pm 8.51(29-38)$ & $Z=-0.21 ; P=0.83$ \\
MDLQ - Patients ${ }^{+}$ & $84.40 \pm 44.52(49-156)$ & $105.60 \pm 55.04(48-187)$ & $Z=-0.52 ; P=0.60$ \\
MDLQ - Caregivers ${ }^{+}$ & $172.00 \pm 22.47(149-197)$ & $143.25 \pm 59.20(111-232)$ & $Z=-0.94 ; P=0.34$ \\
ADL & $42.80 \pm 4.55(38-47)$ & $41.20 \pm 4.09(37-46)$ & $Z=-0.84 ; P=0.40$ \\
Functional Test & $5 \pm 2.00(2-7)$ & $7 \pm 0.71(6-8)$ & $Z=-1.96 ; P=0.04$ \\
\end{tabular}

Data are reported as means $\pm S D$, with the range in parentheses. MMSE $=$ Mini-Mental State Examination; HAM-A = Hamilton Anxiety Scale; MADRAS = Montgomery-Alsberg Depression Rating Scale; $\mathrm{ADL}=$ interview to determine deterioration in daily functioning in dementia; $\mathrm{QOL}$ = Quality of Life Questionnaire; MDLQ = Memory of Daily Living Questionnaire.

+ In this variable, small value or small score means positive impact of treatment.

Z: Wilcoxon test.

Table 3. Results of the neuropsychological tests applied to patients pre- and post-neuropsychlogical rehabilitation (NR).

\begin{tabular}{lccc}
\hline Test & \multicolumn{1}{c}{ Pre-NR } & Post-NR & Statistics \\
\hline FOME & $22.80 \pm 15.72(7-48)$ & $29.60 \pm 21.10(1-60)$ & $Z=-0.94 ; P=0.34$ \\
Logical Memory I & $7.80 \pm 8.50(0-22)$ & $11.60 \pm 8.05(2-24)$ & $Z=-1.05 ; P=0.29$ \\
Logical Memory II & $3.40 \pm 5.64(0-13)$ & $4.80 \pm 9.15(0-21)$ & $Z=0.00 ; P=1.00$ \\
Visual Reproduction I & $15.80 \pm 10.64(0-21)$ & $16.80 \pm 6.98(8-26)$ & $Z=-0.10 ; P=0.91$ \\
Visual Reproduction II & $5.20 \pm 7.26(0-60)$ & $5.60 \pm 6.54(0-16)$ & $Z=-0.33 ; P=0.73$ \\
Recognition Memory Face & $31.40 \pm 4.98(27-38)$ & $31.80 \pm 7.40(25-41)$ & $Z=-0.21 ; P=0.83$ \\
Boston & $40.60 \pm 12.56(26-52)$ & $46.40 \pm 7.80(38-55)$ & $Z=-0.83 ; P=0.40$ \\
Forward Digit Span & $5.00 \pm 1.41(3-7)$ & $5.20 \pm 1.64(4-7)$ & $Z=-0.10 ; P=0.91$ \\
Backward Digit Span & $3.80 \pm 1.79(2-6)$ & $3.60 \pm 1.14(2-5)$ & $Z=-0.21 ; P=0.83$ \\
Verbal Fluency Semantic (animals) & $10.80 \pm 3.56(7-14)$ & $10.60 \pm 9.29(1-26)$ & $Z=-0.53 ; P=0.59$ \\
Verbal Fluency Phonemic & $29.80 \pm 11.82(14-45)$ & $27.00 \pm 7.65(14-33)$ & $Z=-0.52 ; P=0.59$ \\
IQ verbal & $87.20 \pm 19.38(70-107)$ & $97.00 \pm 21.12(80-128)$ & $Z=-1.25 ; P=0.20$ \\
IQ performance & $87.20 \pm 15.58(70-114)$ & $89.20 \pm 15.50(71-107)$ & $Z=-0.31 ; P=0.75$
\end{tabular}

Data are reported as means $\pm S D$, with the range in parentheses. Boston $=$ Boston Naming Test; FOME $=$ Fuld Object Memory Evaluation; $1 \mathrm{Q}=$ intelligence quotient.

Z: Wilcoxon test. 


\section{Discussion}

$\mathrm{AD}$ patients present memory problems in both the storage and retrieval stages causing ADL impairment. It may be possible to reduce these deficits through strategies that use the patient's implicit memory to learn or re-learn information, and training in ADL with external aid.

The results of the present study showed a significant improvement after training only on the Functional Test, and modest improvement in some cognitive tests and psychiatric symptoms. This could be partially explained by the small sample size, the low frequency of NR training sessions. Nevertheless, the ES analyses showed positive results of NR training.

Methodological aspects such as no comparison to a control group (AD patients who were under medication but not under NR training) should be mentioned and are justified by the fact that this was a pilot study with the specific objective (test and scale selection) of evaluating pre- and post-treatment results of implicit and explicit memory techniques and $\mathrm{ADL}$ training.

It is well known that rivastigmine treatment can improve cognitive function and ADL performance in the first three months

Table 4. Effect size of treatment of patients with Alzheimer's disease determined with some scales and tests.

\begin{tabular}{lc}
\hline Scales and tests & Effect size \\
\hline MMSE & 0.11 \\
ADL & 0.35 \\
Functional Test & 1.00 \\
Logical Memory I & 0.44 \\
Logical Memory II & 0.24 \\
Visual Reproduction I & 0.09 \\
Visual Reproduction II & 0.05 \\
Recognition Memory Face & 0.08 \\
FOME & 0.3 \\
\hline
\end{tabular}

MMSE $=$ Mini-Mental State Examination; $\mathrm{ADL}=$ interview to determine deterioration in daily functioning in dementia; FOME = Fuld Object Memory Evaluation. of treatment, with stabilization or a slight decrease after this period $(30,31)$. Since all patients studied had been taking the medication for at least three months before the beginning of NR training, the positive effects observed after training are probably the result of both treatments, including caregiver orientation.

The present results are consistent with others published recently, which showed a more positive clinical effect rather than a statistically significant effect on tests and scales $(10,32,33)$. However, there are only a few controlled studies with specific techniques of implicit and explicit memory and training to minimize memory and ADL deficits in AD. For example, Zanetti et al. (4) conducted a study with 10 mild-moderate AD patients using a procedural memory stimulation program. Five patients were trained in half of the 20 daily activities $1 \mathrm{~h} /$ day every day for 3 weeks, and 5 patients were trained in the other half. There was a significant reduction in time spent to perform the trained procedures compared to the untrained ones. This study indicates that the rehabilitation of ADL through the development of procedural strategies may be effective in mild and moderate $\mathrm{AD}$ patients. Improvement was also present in "not trained" activities, suggesting that functional achievements may be independent of the learning context. Camp and McKitrick (34), after preliminary findings, also suggested that "implicit memorybased intervention is more likely to yield positive results in $\mathrm{AD}$ patients than interventions based on explicit memory".

Studies with NR of memory in AD patients have emphasized the importance of rehabilitation associated with drug treatment. De Vreese et al. (10) divided 24 patients with $\mathrm{AD}$ into 4 groups and compared them: 1) placebo, 2) treatment with AChE-I, 3) neuropsychological rehabilitation, and 4) AChE-I + NR. After 3 months of drug treatment, groups 3 and 4 started NR for a period of 3 months. Patients participated in indi- 
vidual sessions of NR coupled with caregiver training. The results suggested better efficacy of combined treatment (AChE-I + NR), with marked therapeutic effects on cognition, behavior alteration and ADL.

Bottino el al. (35) reported the preliminary results of combined treatment of a group of 6 mild-moderate AD patients for 6 months. They showed stabilization or a small improvement of patient cognitive deficits and ADL by the end of the NR program. The authors suggested that the combined treatment could help stabilization and even result in a reduction of cognitive and functional deficits in $\mathrm{AD}$ patients.

Another interesting study compared both treatments, stimulation of procedural memory with ADL training, and partially spared cognitive function training such as memory, attention and language. Each program consisted of 5-week individual training, 3 days a week, with 45-min sessions per day for mildmoderate AD patients. The study concluded that both $\mathrm{AD}$ groups showed substantial improvement after training in a direct performance measure of everyday functioning. However, the results of neuropsychological tests suggested that ADL training may be more effective than stimulating "residual" cognitive functions (36).

One of the major general problems and criticisms about teaching complex mnemonic strategies to $A D$ patients is that very few people are actually able to apply these strategies to day-to-day problems. Therefore, it is important to use such mnemonic techniques as specific tools to be employed only when the patients need to learn something important and not as a general principle for daily difficulties (37). For this matter, the best solution seems to be the specific training for specific difficulties.
The current study suggests that the assessment of psychiatric symptoms by scales such as the Hamilton Anxiety Scale and Montgomery-Alsberg Depression Rating Scale, cognition and ADL activities by Functional Test and by neuropsychological measures including Wechsler Memory Revised Scale, Wechsler Intelligence Revised Scale, Fuld Object Memory Evaluation, Recognition Memory Face, Boston Naming Test, and Verbal Fluency may be able to identify improvements after NR treatment. The Functional Test used here is also an effective and relevant instrument since it evaluates changes in a more ecological fashion.

The present study also indicates that patients can apply memory techniques and perform simple activities routinely. In addition, improving simple activities promotes important gains in behavior, improves patient independence and minimizes caregiver overload.

Our results support the view that a NR program associated with pharmacological treatment (AChE-I) and caregiver support groups applied to mild AD patients represents a realistic goal to slow down AD cognitive deficits and to reduce the psychiatric symptoms. Weekly stimulation of memory, language and training of $\mathrm{ADL}$ is believed to be of great value in $\mathrm{AD}$ treatment, not only delaying the progress of the disease, but also improving some cognitive functions and ADL.

Non-pharmacological strategies applied to $\mathrm{AD}$ patients and caregiver support groups are important and our data suggest that patients are able to maintain their preserved cognitive functioning for a longer period of time even though $\mathrm{AD}$ is a progressive degenerative disease. 


\section{References}

1. Morris RG \& Kopelman MD (1986). The memory deficits in Alzheimer-type dementia: A review. Quarterly Journal of Experimental Psychology, Special Issue: Human Memory, 38A: 575-602.

2. Clare L, Wilson BA, Carter G, Breen K, Gosses A \& Hodges JR (2000). Intervening with everyday memory problems in dementia of Alzheimer type: an errorless learning approach. Journal of Clinical and Experimental Neuropsychology, 22: 132-146.

3. De Vreese LP, Neri M, Fioravanti M, Belloi L \& Zanetti O (2001). Memory rehabilitation in Alzheimer's disease: a review of progress. International Journal of Geriatric Psychiatry, 16: 794-809.

4. Zanetti O, Binetti G, Magni E, Rozzini L, Bianchetti A \& Trabucchi M (1997). Procedural memory stimulation in Alzheimer's disease: Impact of a training programme. Acta Neurologica Scandinavica, 95: 152-157.

5. Spector A, Orrell M, Davies S \& Woods B (2000). Reminiscence therapy for dementia (Cochrane Review). In: The Cochrane Library. Issue 2. Update Software, Oxford, UK.

6. Spector A, Orrell M, Davies S \& Woods B (2001). Reality orientation for dementia (Cochrane Review). In: The Cochrane Library. Issue 4. Update Software, Oxford, UK.

7. McLellan DL (1991). Functional recovery and the principles of disability medicine. In: Swash M \& Oxbury J (Editors), Clinical Neurology. Churchill Livingstone, London, UK.

8. Clare L \& Woods B (2001). Editorial: A role for cognitive rehabilitation in dementia care. In: Clare L \& Woods B (Editors), Cognitive Rehabilitation in Dementia. Psychology Press, London, UK.

9. Kitwood T (1991). Dementia Reconsidered: The Person Comes First. Open University Press, Buckingham, UK.

10. De Vreese LP, lacono S, Finelli C, Gianelli MV \& Neri M (1998). Enhancement of therapeutic effects of drug treatment in DAT when combined with cognitive retraining? A three month program. Neurobiology of Aging, 19(S4): 212-213.

11. Folstein NF, Folstein SE \& McHugh PR (1975). Mini mental state: a practical method for grading the cognitive state of patients for the clinician. Journal of Psychiatric Research, 12: 189-198.

12. Montgomery SA \& Alsberg M (1979). A new depression scale designed to be sensitive to change. British Journal of Psychiatry, 134: 382-389.

13. Bruss GS, Gruenberg AM, Goldstein RD \& Barber JP (1994). Hamilton Anxiety Rating Scale interview guide: joint interview and testretest methods for interpreter reliability. Psychiatric Research, 53: 191-202.

14. Tuenisse S \& Derix MMA (1999). Interview to determine deterioration in daily functioning in dementia. Tijdscriff voor Gerontologite en Geriatric, 22: 53-59.

15. Sunderland A, Harris JE \& Gleave J (1984). Memory failures in everyday life following severe head injury. Journal of Clinical Neuropsychology, 6: 127-142.

16. Novelli MMPC (2003). Adaptação transcultural da escala de avaliação de qualidade de vida na doença de Alzheimer. Master's thesis in Sciences, Experimental Fisiopathology Area, University of São Paulo, São Paulo, SP, Brazil.

17. Wechsler D (1987). Wechsler Memory Scale - Revised. The Psychological Corporation, New York.

18. Wechsler D (1981). Wechsler Intelligence Scale - Revised. The Psychological Corporation, New York.

19. Fuld PA (1980). Guaranteed stimulus-processing in the evaluation of memory and learning. Cortex, 16: 255-272.

20. Warrington RK (1984). Recognitions Memory Test. NFER-Nelson,
Windsor, UK

21. Goodglass H, Kaplan E \& Barresi B (1998). The Assessment for Aphasia and Related Disorders. 3rd edn. Lippincott Williams \& Wilkins, Baltimore, MD, USA.

22. Spreen A \& Strauss E (1998). A Compendium of Neuropsychological Tests - Administration, Norms and Commentary. 2nd edn. Oxford University, New York.

23. Brucki SM, Malheiros SM, Okamoto IH \& Bertolucci PH (1997). Normative data on verbal fluency test in the animal category in our milieu. Arquivos de Neuro-Psiquiatria, 55: 56-61 (Portuguese).

24. Spreen A \& Benton AL (1969). Neurosensory Center Comprehensive Examination for Aphasia (NCCEA). University of Victoria Neuropsychology Laboratory, Victoria.

25. Goldstein $G$ \& Beers $S$ (1988). Assessment and planning for memory retraining. In: Goldstein G \& Beers S (Editors), Rehabilitation. Plenum, New York.

26. Squire LR \& Kandel ER (1999). Memory - From Mind to Molecules. Scientific American Library, New York.

27. Baddeley AD \& Wilson BA (1994). When implicit learning fails: amnesia and the problem of error elimination. Neuropsychologia, 32: 53-68.

28. Ready RE, Ott BR \& Grace J (2004). Patient versus informant perspectives of quality of life in mild cognitive impairment and Alzheimer's disease. International Journal of Geriatric Psychiatry, 19: 256-265.

29. Rockwood K, Joyce B \& Stolee P (1997). Use of goal attainment scaling in measuring clinically important change in cognitive rehabilitation patients. Journal of Clinical Epidemiology, 50: 581-588.

30. Rösler M, Anand R, Cicin-Sain A, Gauthier S, Agid Y, Dal-Bianco P, Stähelin HB, Hartman R \& Gharabawi M (1999). Efficacy and safety of rivastigmine in patients with Alzheimer's disease: international randomized controlled trial. British Medical Journal, 318: 633-640.

31. Farlow M, Potkin S, Koumaras B, Veach J \& Mirski D (2003). Analysis of outcome in retrieved dropout patients in a Rivastigmine vs placebo, 26 weeks, Alzheimer disease trial. Archives of Neurology, 60: 843-848.

32. Davis RN, Massman PJ \& Doody RS (2001). Cognitive intervention in Alzheimer disease: a randomized placebo-controlled study. Alzheimer Disease and Associated Disorders, 15: 1-9.

33. Heiss WD, Kessler J, Mielke R, Szelies B \& Herholz K (1994). Longterm effects of phosphatidylserine, pyritinol and cognitive training in Alzheimer's disease. A neuropsychological, EEG, and PET investigation. Dementia, 5: 88-98.

34. Camp CJ \& McKitrick LA (1992). Memory intervention in Alzheimer's type dementia populations: methodological and theoretical issues. In: West RL \& Sinnot JD (Editors), Everyday Memory and Ageing: Current Research and Methodology. Spring-Verlag, New York.

35. Bottino CMC, Carvalho IAM, Alvarez AMM, Ávila R, Zukauskas PR, Bustamante SEZ, Andrade FC, Hototian SR, Saffi F \& Camargo CHP (2002). Cognitive rehabilitation in Alzheimer's disease patients: multidisciplinary team report. Arquivos de Neuro-Psiquiatria, 60: 70-79.

36. Farina E, Fioravanti R, Chavari L, Imbornone E, Alberoni M, Pomat G, Pignatti R \& Marinari C (2002). Comparing two programs of cognitive training in Alzheimer's disease: a pilot study. Acta Neurologica Scandinavica, 105: 365-371.

37. Baddeley A (2001). Treating memory problems. In: Baddeley A (Editor), Human Memory. Psychology Press, London, UK. 


\section{Appendix}

Functional Test

Patient's name:

Date of evaluation:

Chores:

1. Receive and take note of a message by phone. Tell the patient: "when the phone rings you should answer it" - "Mr.(s) has a doctor appointment at the hospital next Monday at three o'clock"

a) answers the phone spontaneously

b) says that he/she will take the message spontaneously

c) takes the message before hanging up the phone

d) checks that he/she wrote the message down correctly before hanging up the phone

Observations:

2. Take note of an appointment in the diary. Tell the patient: "I will tell you an appointment and you will take note of this appointment in your diary: "Mr.(s) has a dentist appointment next Wednesday at two o'clock"

a) manages to locate today's date in the diary without any help

b) takes note of the appointment on the right day, that is, the day of the appointment

c) takes note of the complete details of the appointment

Observations:

3. Write a note giving someone a message. Tell the patient: "I will give you a message for you to tell the other person: "Son, the cleaning lady called informing that she will not be coming to work tomorrow"

a) takes note of the message alone

b) takes note of the message completely

Observations: 